\title{
EN TORNO A LA DISPOSICIÓN AFECTIVA Y SU POSIBLE INTERPRETACIÓN REALISTA EN SER Y TIEMPO DE MARTIN HEIDEGGER
}

\author{
ON ATTUNEMENT AND ITS POSSIBLE REALIST INTERPRETATION IN \\ HEIDEGGER'S BEING AND TIME
}

\author{
María Sol Yuan ${ }^{1}$ \\ Universidad Nacional del Litoral - CONICET (Argentina)
}

Recibido: 09-09-2014

Aceptado: 23-12-2014

\begin{abstract}
Resumen: El presente trabajo tiene por tema el análisis de la noción de disposición afectiva (Befindlichkeit) empleada por Martin Heidegger en Ser y Tiempo (1927) y, en particular, en la indagación de la propuesta acerca de una posible lectura realista que se establecería a partir del rol que en este espacio juega el mencionado fenómeno, en tanto reúne en sí uno de los rasgos existenciales (Existenzial) que con mayor claridad denota el hecho de que el mundo "nos afecta» de determinado modo. Es nuestra intención, mostrar la inviabilidad de tal lectura al considerarla en el contexto de la propuesta del autor.
\end{abstract}

Palabras-clave: Heidegger, Realismo, Disposición afectiva, Ser y Tiempo.

\begin{abstract}
This paper studies the notion of 'attunement' (Befindlichkeit) employed in Martin Heidegger's Being and Time (1927). More specifically, it centers in the realistic interpretation of Heidegger, sustained by the view that the moods shows with major clarity the fact that the world affects us in a determinate way. The intention of this task is argues against this possible lecture in the whole context of Being and Time.

Key-words: Heidegger, Realism, Attunement, Being and Time.
\end{abstract}

[1] (msolyuan@hotmail.com) Licenciada en Filosofía por la Universidad Nacional del Litoral (UNL). Doctoranda en Filosofía en la Universidad de Buenos Aires (UBA). Becaria tipo II del Consejo Nacional de Investigaciones Científicas y Técnicas (CONICET). 
Heidegger rechazó ser catalogado en términos realistas o idealistas. Sin embargo, afirmaciones sobre la necesidad de "confiar el descubrimiento primario del mundo al 'mero estado de ánimo'» (SZ §29, 138) ${ }^{2}$, han abierto la posibilidad de realizar una lectura realista a partir de la consideración de la afectividad, resaltando la pasividad del Dasein en este "ser afectado» por las cosas. Así, mientras una postura en torno a la interpretación idealista de Heidegger se basa en el hecho de que la conciencia de los entes está fundada en la comprensión del ser por parte del Dasein, autores como Piotr Hoffman ${ }^{3}$ se detienen en el hecho de que lo que está descubierto siempre se halla de tal modo a partir de un estado de ánimo determinado y estos son autónomos en relación a la comprensión y de ahí, consecuentemente, pueden asegurar un acceso autónomo a lo que nos rodea. ${ }^{4}$ La caracterización del realismo que estos autores están considerando consistiría en algo así como pensar la posibilidad de que los entes existan y mantengan al menos algunas de sus propiedades independientemente de que el sujeto las esté conociendo o percibiendo. Implicaría, en mayor o menor grado, aceptar la autonomía con respecto al Dasein en la constitución de la realidad.

En Heidegger esta pregunta acerca de la determinación de una postura realista es particularmente difícil, ya que debemos sumar el planteo ontológico que en Ser y Tiempo se lleva adelante como fundamento de cualquier investigación óntica, en la cual deberíamos situar diferentes aspectos del fenómeno del conocimiento.

Dentro de un contexto más general, el tema aquí desarrollado se encuadra dentro de los objetivos de determinar la participación de la afectividad en el desvelamiento del mundo, considerando la misma como un elemento irreductible a un costado de la razón. Además, la aclaración del rol que posee la disposición afectiva en nuestro modo de ser en el mundo posee vital relevancia desde que, para Heidegger, la misma constituye un presupuesto y el medio en el que el pensar y obrar se desarrollan, y ello debido a que en la disposición

[2] Heidegger, M.: Sein und Zeit, Max Niemeyer Verlag, Tübingen, 1963. De ahora en adelante: «SZ», seguido del número de parágrafo y página.

[3] Hoffman, P.: "Heidegger and the Problem of Idealism" en Dreyfus \& Wrathall ed.: Heidegger reexamined / Vol. II: Truth, Realism and The History of Being. New York \& London: Routledge, 2002; pp. 319-327.

[4] Estas lecturas en clave idealista y realista han sido tarea emprendida y debatida por intérpretes en su mayoría anglo-americanos. Cf. por ej.: Blattner, W.: "Is Heidegger a Kantian Idealist?" en Dreyfus \& Wrathall ed.: Heidegger Reexamined / Vol. II: Truth, Realism and History of Being. New York, London: Routledge, 2002; pp. 231-248. Olafson, F.: Heidegger and the Philosophy of Mind. New Haven: Yale University Press, 1987; p. 140. Carman, T.: Heidegger's Analytic / Interpretation, Discourse and Authenticity in Being and Time. New York: Cambridge University Press, 2003. Dreyfus, H. \& Spinosa, Ch.: "Coping with Things-in-themselves: A Practice-Based Phenomenological Argument for Realism” en Dreyfus \& Wrathall ed.: Heidegger Reexamined vol. II / Truth, Realism and the History of Being. London \& New York: Routledge, 2002; pp. 249-278.

THÉMATA. Revista de Filosofía, $\mathrm{N}^{\circ} 51$ enero-junio (2015) pp.: 383-400 doi: 10.12795/themata.2015.i51.20 
afectiva tiene lugar una apertura del mundo en sentido ontológico, y no un sencillo comportamiento entre otros.

Tal postura se distancia de las conclusiones en torno al rol de los estados de ánimo de gran parte de la tradición filosófica antigua y moderna, donde el lado racional (a veces sumado a la percepción de los sentidos) era el único encargado de las funciones cognitivas del ser humano y opuesto al lado emocional o afectivo. ${ }^{5}$

Pero Heidegger también toma distancia dentro de la Fenomenología, del equilibrio propuesto por Husserl entre lo que este autor consideró dos formas o modos de conciencia: la percepción y la afectividad (a la que debemos añadir, en realidad, un tercer modo: la volición). La propuesta del maestro parece tomar otros matices en Heidegger mostrando, por un lado, un equilibrio entre la comprensión y la disposición afectiva (estipulado en el carácter co-originario de estos fundamentos). Por otro lado, y de modo simultáneo, Heidegger establece la primacía de estos existenciales en relación a los actos intencionales en general y al conocimiento teórico inherente a los mismos.

Estructuraremos la exposición del siguiente modo: en primer lugar, señalaremos muy brevemente qué entiende Heidegger por disposición afectiva en Ser y Tiempo. En segundo lugar, realizaremos una descripción de una lectura realista de la disposición afectiva. Luego, abordaremos las dificultades que tal lectura presenta a partir de la interposición de tres interrogantes:

1) Sobre la posibilidad de una teoría del conocimiento basada en los estados de ánimo

2) Objeción basada en la co-originariedad

3) Objeción basada en la preeminencia del futuro en la temporalidad (Zeitlichkeit) propia del Dasein.

Luego de estas indagaciones, concluiremos que resulta difícil sostener una postura realista de acceso a los entes mundanos basados en la simple disposición afectiva, al menos si intentamos no separarla del resto de los elementos que acompañan el análisis de la misma en Ser y Tiempo.

Antes de continuar, resulta necesaria una aclaración. Situar de un modo adecuado el rol que cumple la disposición afectiva en relación a los estados o procesos cognitivos del Dasein, implicaría un detallado recorrido que pasaría por la caracterización del conocimiento apofántico, su raíz práctico-vital y cotidiana del Dasein, hasta llegar al análisis propiamente existencial en el que

[5] Como excepción a esta generalización, encontramos la interpretación de Aristóteles realizada por Martha Nussbaum en su libro Love's Knowledge. Cf. Nussbaum, M. C.: Love's Knowledge / Essays on Philosophy and Literature. New York: Oxford University Press, 1990; pp. 261-286. Heidegger, por su parte, consideró a Aristóteles como el primer investigador sistemático de los afectos y seguramente el más eminente hasta su tiempo: «la interpretación ontológica fundamental de lo afectivo no ha podido dar un solo paso hacia adelante digno de mención después de Aristóteles» (SZ §29, 139).

THÉMATA. Revista de Filosofía, №51 enero-junio (2015) pp.: 383-400 doi: 10.12795/themata.2015.i51.20 
hallamos el fundamento ontológico de los temples anímicos. Lamentablemente, una tarea que comenzara por allí agotaría el espacio del trabajo y dilataría la descripción del núcleo temático del mismo. Nos centraremos, por lo tanto, en el análisis de la disposición afectiva y dejaremos limitados los restantes desarrollos a lo estrictamente necesario según nuestros actuales intereses.

\section{Caracterización de la disposición afectiva}

Una lectura realista de Ser y Tiempo podría justificarse gracias a la íntima conexión que Heidegger establece entre la noción de facticidad y el carácter ontológico del Dasein que puede traducirse como «disposición afectiva» (Befindlichkeit) - SZ §12. El suelo fenoménico que constituye el punto de partida para el análisis de la disposición afectiva es el hecho de que siempre y cada vez nos encontramos vitalmente determinados por estados de ánimo (Stimmungen), los cuales muestran el modo como uno "se encuentra" en el mundo, "cómo le va a uno» («wie einem ist und wird» - SZ §29, 134). Por un lado, este fenómeno muestra la situación o localización del Dasein, es decir, abre el «espacio de juego» (Spielraum) donde se encuentra. Por otro lado, indica que esta apertura siempre se realiza de un modo determinado. ${ }^{6}$

Podemos señalar como primera característica entonces el fenómeno de apertura del Dasein a sí mismo en su condición de arrojado. Este carácter, lejos de constituir un proceso interno, nos lleva a la indisoluble unión entre el Dasein y el mundo, desde el punto de vista de que el Dasein es siempre su Ahí (Da). Lo que muestran los estados de ánimo es que el Dasein «es y tiene que ser» siempre y cada vez de un modo determinado, es decir, ponen en evidencia la existencia del Dasein como factum: el Dasein es siempre abriendo su mundo. ${ }^{7}$

El encontrarse a sí mismo dispuesto a ser afectado de determinado modo por el mundo no debe entenderse como un proceso de interioridad en el cual adquiero autoconciencia de mis estados afectivos. No hay una instancia auto-perceptiva de un yo aislado en este planteo. Cualquier conocimiento de sí, por otro lado, implicaría ya una determinada disposición afectiva previa que

[6] Cf. Inwood M.: A Heidegger Dictionary / The Blackwell Philosopher Dictionaries. Oxford: Blackwell Publishers, 1999, p. 31.

[7] Esta «enigmaticidad» refiere a una facticidad en un sentido preciso. En primer lugar, muestra al Dasein el hecho de que es, lo deja ante la inexorable enigmaticidad de que es, aunque no sepa de dónde ni hacia dónde. Según Stephen Crowell, quien dedica su artículo "Facticity and Transcendental Philosophy" a este asunto, la facticidad indica al Dasein que «en relación a su auto-comprensión ningún conocimiento suyo - incluso su herencia histórica - puede ser asumida como el fundamento de su identidad». En segundo lugar, trae al Dasein ante el «que es» pero no lo hace señalando meramente un hecho sino, antes bien, un "tener que ser [zu sein]». Esto no implica que el Dasein deba entenderse como un ser necesario, por el contrario, este debe deliberar sobre el ser que, en su existencia, tiene que ser. Cf. Crowell, S.: "Facticity and Transcendental Philosophy" en Malpas, J. ed.: From Kant to Davidson. London, New York: Routledge, 2003; pp. 100-121, p. 115.

THÉMATA. Revista de Filosofía, No 51 enero-junio (2015) pp.: 383-400 doi: 10.12795/themata.2015.i51.20 
predisponga de dicho modo mi comprensión. Es en este sentido que Heidegger afirma que «jamás seremos dueños de un estado de ánimo sin otro estado de ánimo» (SZ §29, 136).

Entramos de este modo en las dos características constitutivas restantes de la Befindlichkeit que refieren al modo de ser su Ahí por parte del Dasein. El segundo carácter ontológico muestra cómo la Befindlichkeit determina la apertura del mundo en su totalidad (SZ §29, 137). Esto es, el sí-mismo no es nada semejante a un "yo» puntual y aislado más o menos cerrado sobre sí y carente de mundo. Se trata, por el contrario, del sí-mismo concreto ya siempre vuelto hacia las cosas y hacia los otros con los que comparte el mismo «modo de ser». Lo que el «encontrarse» pone, por tanto, de manifiesto incluye estas dos dimensiones en su inescindible unidad: cómo se encuentra el yo en la ejecución de la totalidad de sus referencias mundanas y cómo se le muestran los entes del mundo a partir de una peculiar tonalidad emotivo-vital. Precisamente porque en Heidegger la relación entre Dasein y mundo no es la de un mero sujeto enfrentado a un objeto o la de un "dentro" que se vincula con un «fuera», tampoco valen estas polaridades para pensar la fuente y alcance de la disposición afectiva. Heidegger dirá por ello que «el estado de ánimo nos sobreviene» (SZ $\S 29,136)$, y ello debido a que se sostiene en el fenómeno unitario del ser-en-elmundo del Dasein.

Por último, el tercer carácter esencial que marca el encontrarse de un modo afectivamente dispuesto a «algo» muestra cómo, al posibilitar la apertura del Dasein y del mundo, deja comparecer de un modo simultáneo a los entes intramundanos, y de un modo correlativo, permite al Dasein ser afectado por aquello que comparece.

La disposición afectiva muestra con toda claridad cómo la ocupación constante con lo que nos rodea de un modo inmediato tiene el carácter de «ser concernido" (betroffenwerden). Las cosas adquieren sentido para el Dasein porque éste ya se encuentra dirigido previamente hacia ellas. El sujeto humano puede ser afectado por lo dado tan sólo porque él mismo no es un ser cerrado, sino que se encuentra siempre en la apertura de sentido de su mundo y consignado al trato con lo intramundano, todo ello de una manera determinada por los diferentes temples anímicos.

\section{Posible lectura realista de la relación entre el Dasein y el mun- do a partir de la facticidad mostrada en los estados de ánimo}

Esta última descripción de la disposición afectiva constituye, de un modo preeminente, una apertura a la posibilidad de interpretar un acceso a lo que nos rodea en clave realista, desde que el «estar-consignado» implica una cierta dependencia respecto de los entes y el mundo. Según Heidegger, es gracias a esta característica que resulta necesario en primer lugar y desde el pun- 
to de vista ontológico, «confiar el descubrimiento primario del mundo al 'mero estado de ánimo'» (SZ §29, 138), aunque esto implique reconocer de un modo conjunto que no hay una uniformidad constante y presente del mundo, sino que este se manifiesta de modos múltiples a través de las posibilidades planteadas por nuestro estado anímico. Incluso la mirada contemplativa lleva a cabo su pretensión de ver aquello constante e invariable, presente «ante los ojos», tan sólo desde un temple anímico que lo lleva a esta posibilidad.

El estar templado por los entes, determinados en cierta medida por ellos, implica no sólo un estar acordados con las cosas, sino estarlo también bajo alguna determinación de ellos: somos determinados, en el sentido de afectados, siempre de algún modo por el mundo, y esto es lo que marca el carácter en primera instancia no-psicologista del análisis de la disposición afectiva. En el curso del semestre de verano de 1925, Heidegger explicita:

\footnotetext{
Este carácter de estar al descubierto en la disposición afectiva está conectada con el ser-en-el-mundo como tal... tal que en todo lo que hacemos y donde tratamos con las cosas, somos en algún sentido -como decimos -'afectados' (PGZ §28-a, 352). ${ }^{8}$
}

Este ser afectados no implica una tematización explícita de los entes con los que acordamos en cada temple anímico, sino que se trata de un encontrarse a sí mismo en el mundo, donde el «sí mismo» no se refiere a un yo necesariamente descubierto y temáticamente consciente (PGZ §28-a, 351) y donde el «Ahí» se descubre también de un modo a-temático (PGZ §28-a, 354).

Parte de los argumentos señalados aquí han sido retomados por el intérprete Piotr Hoffman en su artículo "Heidegger and the Problem of Idealism"9 a fin de sostener la hipótesis de que se puede realizar una lectura realista. Su trabajo parte observando que la mayoría de las interpretaciones idealistas surgen de la consideración exclusiva del rol de la comprensión en la descripción del Dasein olvidando así su carácter arrojado manifiesto en la disposición afectiva. Sin embargo, esto no significa que, aun reconociendo a aquel existencial su exacta participación en la aperturidad del Dasein, sea posible de hecho lograr librarnos de la afirmación de que los entes sean inevitablemente inteligibles a partir de cierta comprensión del ser.

Llegado este punto, y a fin de argumentar a favor de su posición, Hoffman abandona los textos de Ser y Tiempo para presentar lo que considera un modo alternativo al basado en la comprensión a fin de descubrir los entes, apoyándose para ello en algunos pasajes correspondientes al curso de verano de 1928 dictado por Heidegger, editado bajo el título: Metaphysische Anfangsgründe der Logik im Ausgang von Leibniz. ${ }^{10}$

[8] Heidegger, M.: Prolegomena zur Geschichte des Zeitsbegriffs, Gesamtausgabe 20, Vittorio Klostermann, Frankfurt am Main, 1979. De ahora en adelante: PGZ, seguido del número de parágrafo y número de página.

[9] Op. cit.

[10] Heidegger, M.: Metaphysische Anfangsgründe der Logik im Ausgang von Leibniz, Gesamtaus-

THÉMATA. Revista de Filosofía, No51 enero-junio (2015) pp.: 383-400

doi: 10.12795/themata.2015.i51.20 


\section{En torno a la disposición afectiva y su posible interpretación realista en Ser y Tiempo de Martin Heidegger}

En este curso Heidegger presenta dos modos posibles de descubrir a los entes a partir de dos modos de conocimiento: el primero, el conocimiento del ser, el cual se basa en la existencia del Dasein, esto es, en términos de la proyección comprensora del mismo. El segundo es el conocimiento de «lo sobrecogedor» (Übermächtige) ${ }^{11}$, basado en la condición de arrojado del Dasein, esto es, a través de nuestra disposición afectiva expuesta cada vez por un estado de ánimo. Encontramos en los textos del seminario citas de apoyo a esta lectura:

Afirmamos: la filosofía como filosofía primera tiene, por tanto, un doble carácter, es ciencia del ser y ciencia de lo sobrecogedor [Übermächtige] (Este doble carácter corresponde a la dualidad de existencia y condición de arrojado) - MAL, p. 13.

Heidegger se encuentra aquí definiendo a la filosofía a partir del comienzo gigantesco, aunque "inacabado» e «imperfecto», que Aristóteles proporcionó. Así, habría que contemplar a la filosofía primera como la ciencia que «investiga el ente en tanto que ente", cuyo tema es precisamente aquello que hace al ente el ente que es: el ser. Una disciplina así constituida se diferenciaría de las ciencias que estudian una región del ente o el conjunto de todas estas esferas.

Pero a la vez, la filosofía "auténtica" debe referirse a los fundamentos de lo sobrecogedor que se manifiesta en los entes, esto también a partir de la lectura que Heidegger hace de Aristóteles, quien define a la filosofía como theología y afirma que «lo divino» (tó theíon) se halla en la naturaleza. ${ }^{12} \mathrm{De}$ modo similar, Heidegger propone que la filosofía sea también una ciencia de lo Übermächtige, de lo divino, lo sobrecogedor, lo superpoderoso; que no es otra cosa que una consideración del kósmos:

tò theion significa: el ente sin más -el cielo: lo omniabarcante y lo dominante, aquello bajo lo cual y en lo cual estamos arrojados [geworfen], aquello que nos sorprende [benommen] y nos deja sin respiración, lo sobrecogedor [Übermächtige] (MAL, p. 13).

gabe 26. Frankfurt am Main: Vittorio Klostermann, 1978. De ahora en adelante: MAL. El motivo para tal inclusión se debe, no sólo al hecho de hacer justicia a la postura de este intérprete en el artículo trabajado sino también, y fundamentalmente, a que los aportes de Heidegger en este seminario se encuentran dentro del círculo cercano a la edición de Ser y Tiempo por lo que pueden resultar potencialmente pertinentes. Cabe aclarar, además, que la traducción y referencias bibliográficas que Hoffman trabaja en relación a este tomo de las obras completas es la siguiente: Heidegger, M.: The Metaphyscal Foundations of Logic, trad. M. Heim. Bloomington: Indiana University Press, 1984.

[11] El término empleado por Hoffman para «Übermächtige» es «overwhelming». Hemos elegido traducir la expresión por «lo sobrecogedor», por resultar más afín a nuestra línea interpretativa actual y continuar fiel a la definición dada por el autor alemán sobre la noción: «aquello que nos sorprende y nos deja sin respiración» (MAL, p. 13). García Norro, en su traducción del Seminario en cuestión, elige el término «lo superpoderoso», en una traducción más literal surgida del adjetivo «mächtig»: poderoso, potente, pujante. Cf. . Heidegger, M.: Principios metafísicos de la lógica; trad. Juan José García Norro. Madrid: ed. Síntesis, 2009; p. 21.

[12] La lectura de Aristóteles en la que Heidegger se basa en MAL, pp. 11-14 para estas dos definiciones de filosofía es, fundamentalmente: Metafísica E (1026 a18 - a31).

THÉMATA. Revista de Filosofía, Nº51 enero-junio (2015) pp.: 383-400

doi: 10.12795/themata.2015.i51.20 
Es importante resaltar, además, que según Hoffman esta distinción entre un conocimiento basado en la comprensión del ser y un conocimiento de lo sobrecogedor, del ente mismo, ya se encontraría presente en Ser y Tiempo a partir de las diferencias que en esta obra se dan entre la existencia y la condición de arrojado. Aún más, la cita de Ser y Tiempo que afirma que «...el estado de ánimo pone al Dasein ante el «qué [es]» de su Ahí, que con inexorable enigmaticidad fija en él su mirada» (SZ §29, 136) implicaría afirmar precisamente que los estados de ánimo nos descubrirían la realidad de los entes en tanto fenómeno que «nos sobrepasa» (overwhelming us). "Ahí» significa, en la cita inmediatamente anterior, ahí en medio de los entes: el Dasein se encuentra siempre afectivamente abierto de determinado modo. Por lo tanto, los entes también son descubiertos afectivamente (SZ $\S 29,134)$ y la «inexorable enigmaticidad» es lo que nos sobrecoge, en un rechazo por parte de Heidegger a justificaciones y explicaciones racionales al respecto.

Hoffman recurre al temple de ánimo fundamental que es la angustia como ejemplo de esta distinción. Desde que en la angustia el contexto completo de comprensibilidad colapsa, los entes son ahora desnudados de su significado doméstico, mundano, y el Dasein puede descubrirlos en su radical otredad. Y esto, sin embargo, no implica que la comprensión como tal no exista en la temporalidad propia de la angustia, aunque esté completamente determinada por el estado de ánimo. ${ }^{13}$ La comprensión y la disposición afectiva pueden ser co-originarios, pero a menos que nuestros modos afectivos de descubrir el mundo tengan alguna autonomía, «no podrían distinguirse uno de otro». ${ }^{14}$ Esta afirmación, a simple vista interesante, no resulta un razonamiento necesario: el hecho de que no sean autónomos no significa que sean equivalentes.

Resulta preciso agregar que, aun planteando la autonomía, no demostrada por Hoffman, entre el conocimiento del ser y el conocimiento de lo sobrecogedor (que según lo dicho es el conocimiento del ente sin más), no resolvería esto los inconvenientes a los que se enfrenta la conclusión realista del intérprete en relación a los aportes de la disposición afectiva en Ser y Tiempo. Y es que Heidegger aporta citas en los Metaphysische Anfangsgründe der Logik que nos impiden llegar a la conclusión de Hoffman. Tal es, por ejemplo, la siguiente afirmación:

... el comprender dirigido a él [el ser] es lo primero, en tanto que es el comprender de lo que viene antes de todo lo demás, lo que es anterior, prius, a todo lo otro, a saber: cada ente individual. Pero lo anterior al ente individual es el ser, pues es aquello que ya previamente es entendido, antes de que algo como el ente pueda surgir de cualquier modo en cualquier lugar (MAL, p. 16).

Queda entonces por investigar lo que implica la co-originariedad entre la comprensión y la disposición afectiva, es decir, si su autonomía es a la vez una independencia al punto de permitir accesos separados al mundo y si

[13] Cf. Hoffman, op. cit., p. 324.

[14] Ibídem, p. 325.

THÉMATA. Revista de Filosofía, No 51 enero-junio (2015) pp.: 383-400 doi: 10.12795/themata.2015.i51.20 
su fundamentación no deja lugar a ninguna preeminencia en sus momentos constitutivos, cuestión a desarrollar a partir de la lectura de la temporalidad del Dasein. A esto dedicaremos la segunda y tercera objeción de la parte a continuación.

El primer apartado, que constituye más una aclaración que una objeción, se vincula, por otro lado, con la afirmación de Hoffman de que los estados de ánimo (a través de un conocimiento de lo sobrecogedor) pueden brindarnos un acceso cognitivo, además de autónomo, a los entes. Este punto puede situarse dentro de la cuestión más general acerca de qué es lo que develan los estados de ánimo y si el modo en que lo hacen implica ya un conocimiento de los entes.

\section{Sobre el posible carácter epistémico de la disposición afectiva}

La primera aclaración que deseamos realizar en este apartado surge de las propias palabras de Heidegger en torno al realismo y al idealismo en Ser $y$ Tiempo, a las cuales ve como teorías epistemológicas deficientes surgidas de una concepción representacional del conocimiento que implica una separación tajante entre el sujeto y el objeto, entre un ente ideal y algo que "está realmente ahí» (SZ §44-a, 216-218), y que debe vincularse, también, a la ignorancia del sentido de verdad como «desocultación» en tanto fundamento de la noción de verdad proposicional.

A esto debemos añadir otra crítica complementaria fundamental, y es que tanto el realismo como el idealismo, entendidos como posiciones propias de las teorías del conocimiento, no se aplicaron a la tematización del modo de ser del Dasein (Analítica existencial), por lo cual las tentativas de solución del «problema de la realidad» no llegaron nunca a una conclusión satisfactoria. ${ }^{15}$

Este señalamiento nos previene en un primer sentido: no debemos intentar posicionar a Heidegger en conexión con una lectura realista (tampoco idealista) dentro de parámetros que tengan que ver exclusivamente con el conocimiento teórico de los entes intramundanos, mucho menos si nos manejamos en términos únicamente psicologistas, interioristas, privados, representacionales.

Por lo tanto, de poder decir algo en relación a cómo los temples anímicos descubren lo que nos rodea, debe ser en el marco de referencia ontológico y no en primera instancia epistémico o gnoseológico. El que debamos confiar el descubrimiento primario del mundo a nuestros estados de ánimo no implica, entonces, un sentido cognitivo «fuerte», ya que lo que la disposición afectiva pone de relieve posee un carácter ante todo no-intencional o anterior al desvelamiento intencional del ente intramundano. ${ }^{16}$ En todo caso, nos encontraríamos

[15] SZ §43-a, 206-208.

[16] Si bien Heidegger no hace uso del término «intencionalidad» en Ser y Tiempo dentro del

THÉMATA. Revista de Filosofía, Nº51 enero-junio (2015) pp.: 383-400

doi: 10.12795/themata.2015.i51.20 
en un plano anterior a dicho momento de determinación o "acceso objetivo" a las propiedades de las cosas. Aunque el conocimiento teórico sólo pueda ser llevado adelante desde un temple anímico particular, el mismo implica además el actuar del resto de la estructura de aperturidad del ser-en-el-mundo como condición de posibilidad. Esta apertura es, según las aclaraciones del propio autor, anteriores a todo conocer y querer (SZ §29,136).

Parte de la cuestión depende, en cierta medida, de qué sentido le estemos dando a la noción de 'conocimiento'. Hemos considerado aquí a la idea de conocimiento en tanto conocimiento teórico en un sentido heideggeriano, es decir, un conocimiento enunciativo cuyo objeto está ahí ante-la-mano (vorhanden) o ante los ojos. Ahora bien, esto no imposibilita que podamos preguntarnos si la disposición afectiva tiene realmente alguna relevancia en relación a los procesos cognitivos, más aún en cuanto estos son considerados un modo de ocupación por parte del Dasein, insertos en la estructura total del cuidado que lo caracteriza. Debemos inclinarnos por una respuesta afirmativa: los estados de ánimo constituyen un aspecto del modo en que develamos el mundo. Los estados de ánimo nos abren o descubren las cosas de nuestro entorno como concerniendo al Dasein en un modo particular -aburrido, divertido, indiferente, etc. A su vez, el Dasein está abierto al mundo como algo que puede afectarlo.

Autores como Stephen Mulhall ${ }^{17}$ sostienen, basándose en estos aportes de Heidegger, una posición de apoyo a la posibilidad de desarrollar dentro del campo de la filosofía una epistemología de los estados de ánimo. Lo que posibilita esta lectura es que, dentro del planteo heideggeriano, temples anímicos como el miedo y la angustia poseen un "aspecto subjetivo» y un "aspecto objetivo», lo que nosotros señalamos como el carácter revelador de los estados de ánimo en relación al Dasein y en relación al mundo.

Ahora bien, la dificultad con la que esta descripción nos enfrenta es que, en la angustia, el «ante-qué» (Wovor) de la misma se encuentra indeterminado, no se trata de un ente intramundano determinado o siquiera determinable. Tampoco hay una "zona» propia para este temple anímico y lo amenazante no proviene ni "de aquí» ni "de allí», sino que los entes se muestran carentes de significatividad. Esto es, no se trata de que en la angustia los entes desaparezcan, sino que comparecen sin condición respectiva y en "una vacía inexorabilidad» (SZ §68-b, 343). Heidegger añade que en este estado de ánimo el Dasein se angustia ante sí mismo.

Una lectura sobre la relevancia de la angustia en tanto temple anímico

contexto de análisis de la trascendencia propia del Dasein, podemos comprender que si bien hay un «tender» o «apuntar» hacia contenidos, el mismo no está determinado ni remite a una característica constitutiva de la conciencia.

[17] Mulhall, S.: "Can There be an Epistemology of Moods?" en Dreyfus, Hubert L. \& Wrathall, Mark A.: Heidegger Reexamined vol. 4 / Language and the Critique of Subjectivity. New York, London: Routledge, 2002, p. 33 a 52.

THÉMATA. Revista de Filosofía, No 51 enero-junio (2015) pp.: 383-400 doi: 10.12795/themata.2015.i51.20 
revelador de lo que nos rodea implica entender que la angustia, podríamos decir, carece de objeto entendido como algo específico dentro del mundo, y no podemos reaccionar por lo tanto ante circunstancias específicas como en el caso del miedo (por ejemplo, alejándonos corriendo). Lo que nos oprime «es el mundo en cuanto tal» o mejor, nuestro propio ser-en-el-mundo. A esto se refiere Heidegger cuando afirma que en la angustia el «ante-qué» y «por-qué» (Worum) de la misma coinciden, algo que Heidegger denomina «identidad existencial del abrir y lo abierto» y en la cual radica el carácter eminente o fundamental de este estado de ánimo (SZ §40, 188; §68-b, 342). Si lo que angustia es, entonces, la simple posibilidad del ente en cuanto tal, esto mismo posibilita la revelación del mundo en su mundaneidad (SZ §40, 187), un mundo inherente al ser del Dasein.

Vemos entonces que el temple anímico expuesto por la angustia logra desdibujar los límites entre un plano objetivo y subjetivo aunque no por ello esta explicación ontológica carece, nuevamente, de relevancia en función de nuestros intereses. Según Mulhall, la angustia «elucida la relativa autonomía del mundo», ${ }^{18}$ en tanto esta singularización del Dasein hace patente lo desazonado (unheimlich) de su modo de ser, es decir, que el Dasein reconoce al mundo como lo extraño, aquello que está fuera de nosotros y en lo cual nunca nos encontramos de modo pleno.

Por otro lado, «la angustia nos enseña que el mundo responde a nuestras concepciones de él.» Así, cuando nos angustiamos el mundo se nos vuelve hostil y extraño mientras que, de no realizarse la amenaza de la angustia, el Dasein se siente seguro encubriendo su situación, un "estar-en-casa» (Zuhause-sein) de la familiaridad cotidiana del trato con los entes. Mulhall concluye estos argumentos con las siguientes palabras:

...de acuerdo con la epistemología de Heidegger acerca de la angustia, la externalidad del mundo debe ser comprendida como su capacidad no exhaustiva de ser todos los modos que nuestros estados de ánimo nos dice que puede ser -su capacidad de ser separado de nosotros y a la vez parte de nosotros. ${ }^{19}$

Habiendo establecido el carácter necesario de los temples anímicos en el desvelamiento del mundo, y aún más, la mutua afección que se establece entre los estados de ánimo y el mundo, indagaremos a continuación si los mismos son suficientes o si la aperturidad, tal como señala Heidegger, requiere a la vez de la comprensión del ser y de proyectos por parte del Dasein.

[18] Ibídem, p. 47.

[19] Ídem.

THÉMATA. Revista de Filosofía, Nº51 enero-junio (2015) pp.: 383-400 doi: 10.12795/themata.2015.151.20 


\section{La co-originariedad de la comprensión y la disposición afectiva}

La reiterada afirmación de que la comprensión y la disposición afectiva, junto con la discursividad que las articula, forman parte de una estructura que marca la aperturidad del Dasein, nos enfrenta a algunas dudas y dificultades a la hora de intentar establecer una lectura realista como la expuesta. En particular, y como requerimiento de la misma, nos enfrentamos a la idea de que los estados de ánimo o temples anímicos pueden proporcionar un acceso independiente a los entes. Hoffman afirma que pueden brindar un acceso "autónomo» a ellos, pero creemos que el uso de este término se presta a confusión.

En tal sentido, si bien los temples anímicos son desarrollados por Heidegger de tal modo que su tematización se plantea precisa y diferenciada del existencial de la comprensión y en este sentido podemos decir que tienen su propia caracterización y su propia "tarea», resulta imposible considerar a estos dos momentos estructurales brindando, cada uno por su lado, un acceso total al mundo.

La comprensión "abre" (erschliesst) y mantiene en estado de apertura los entes y, de alguna manera, lo ente en total (sí-mismo, cosas, demás hombres), siendo ésta una "acción originaria" (Ur-handlung) fundante de todas las demás (cognoscitivas, volitivas, prácticas, etc.). Tal como se aclara en Von Wesen des Grundes, ${ }^{20}$ la apertura tiene lugar, pues, como "proyección» de un horizonte de posibilidades que el sujeto pone ante sí como indicación de "ante qué entes y cómo puede actuar» (WG, p. 157).

Ahora bien, este proyecto no es una capacidad de generar sentido exclusivamente desde sí como comienzo absoluto, sino que tiene lugar necesariamente a partir de la situación fáctica en que se encuentra el existir, la cual al mismo tiempo posibilita y limita la actividad proyectiva. El proyecto es, así, siempre un "proyecto arrojado», se da y supone siempre la propia implicación fáctica con aquel círculo de entes con los que se halla vitalmente vinculada. Y es precisamente esta condición fáctica la que, como se vio, se expresa en la disposición afectiva.

Así, afectividad y comprensión se informan e implican de modo recíproco como momentos inseparables que co-constituyen la finitud del existir. A esta relación que se advierte entre ambas estructuras ontológicas Heidegger la llama "co-originariedad» (Gleichursprünglichkeit - SZ §28, 131). La misma implica que sus elementos constitutivos se dan de un modo simultáneo en el Dasein y que la interpretación de cada uno de los fenómenos que así se conforman, consecuentemente, debe ser realizada en correlación con el resto de los elementos participantes de la estructura. En otras palabras, se trata de fenómenos no separables, y por lo tanto no autónomos, en este sentido.

[20] Heidegger, M.: Vom Wesen des Grundes [WG], en Wegmarken, Gesamtausgabe 9. Frankfurt am Main: Vittorio Klostermann, 1976.

THÉMATA. Revista de Filosofía, N ${ }^{\circ} 51$ enero-junio (2015) pp.: 383-400 doi: 10.12795/themata.2015.i51.20 
Sin embargo, esta co-originariedad debe ser entendida de un modo complementario en una dirección inversa a la recién señalada: no sólo no se trata de fenómenos separables sino que, además, ninguno de los elementos que constituyen una estructura co-originaria puede ser deducido del resto. En este sentido podemos dar contexto a una idea de "autonomía» dentro de la co-originariedad: los fenómenos no pueden ser derivados unos de otros en su interior. Este sentido agrega significado preciso a la noción de autonomía aquí en juego, pero no coincide con el significado que Hoffman otorga a la expresión, ya que los estados de ánimo y la comprensión no son autónomos en el primer sentido señalado, sino sólo en el segundo.

A partir de un planteo de tales características se hace fácilmente comprensible la negación del propio Heidegger a cualquier intento de calificar su propio pensamiento en términos de idealismo y/o realismo. 'Disposición afectiva' y 'comprensión' son "caracteres ontológicos constitutivos» que en su multiplicidad aparecen de modo simultáneo e interrelacionados en la constitución misma del ser-en-el-mundo y que se encuentran, por lo tanto, en un mismo nivel de fundamentación. No son parte de un compositum que puede o no formarse.

Por lo tanto, si bien la disposición afectiva y la comprensión poseen su propia «función» en la aperturidad del Dasein, esto no significa que su autonomía llegue al punto de poder explicar el todo de esta estructura unitaria a partir de uno u otro elemento. Intentar hacerlo, según Heidegger, sería caer es esa «tendencia metodológica incontrolada a buscar el origen de todas y cada una de las cosas en un 'primer principio' elemental» (SZ §28, 132).

Resta tratar, por último, una tercera cuestión relativa al modo de vínculo que se establece entre estos dos existenciales, a saber, la posibilidad de considerar a alguno de ellos preeminente en relación al otro.

\section{La temporalidad (Zeitlichkeit) del Dasein y los éxtasis de la disposición afectiva}

El cuidado (Sorge), cuya repetida fórmula que mienta el todo estructural del ser del Dasein, se define como «un anticiparse-a-sí-estando-ya-en-(el-mundo-) en-medio-de (el ente que comparece dentro del mundo» (SZ §41, 192). ${ }^{21}$ Así, el Dasein es en el modo de haberse anticipado ya siempre a sí mismo, proyectando sus propias posibilidades, lo cual señala tres momentos estructurales: 1) el proyectar anticipativo de las propias posibilidades por parte del Dasein, que alude al comprender existencial; 2) el «estar-ya»

[21] Esta traducción, debida a Rivera (op. cit., p. 193), tiene marcadas diferencias con la traducción española realizada por Gaos (op. cit., p. 217). En alemán: «Sich-vorweg-schon-sein-in (der Welt) als Sein-bei (innerweltich begegnendem Seiendem).»

THÉMATA. Revista de Filosofía, No51 enero-junio (2015) pp.: 383-400 doi: 10.12795/themata.2015.i51.20 
arrojado en el mundo que marca la facticidad del Dasein y que se vincula con la disposición afectiva; y 3) un "estar-junto» a los entes a los cuales el Dasein se halla consignado de modos diversos.

Estos tres elementos muestran claramente, como el propio Heidegger se encarga de aclarar más adelante, una significación temporal en la constitución ontológica del Dasein que constituye el cuidado, mostrando una unidad de temporalidad (Zeitlichkeit) conformada por tres éxtasis, direcciones en las que el Dasein "sale de sí» en un sentido temporal, a saber: el futuro (Zukunft), el «haber-sido» (Gewesenheit) y la presentación o presente (Gegenwart), respectivamente. Estas expresiones no deben ser entendidas nunca como una sucesión de «ahoras» sin comienzo ni fin (lo que Heidegger denomina "concepción vulgar» del tiempo) sino, en relación al futuro, como un venir a sí mismo por parte del Dasein que se opone a la idea de futuro como un "todavía no»; en el segundo caso, como lo que aún tiene incidencia sobre nuestro destino como algo que le permite al Dasein "ser como ya siempre era", es decir, asumir la condición de arrojado y, por último, el éxtasis presente que abre la situación del Ahí: «Futuro, haber-sido y presente, muestran los caracteres fenoménicos del «hacia-sí» [«Auf-sich-zu»], del «de-vuelta a» [«Züruck auf»] y del «hacer comparecer algo» ["Begegnenlassen von»]» (SZ §65, 328-329). El sentido ontológico último del ser del Dasein, el cuidado, reside entonces en su «temporalidad».

Ahora bien, dentro de la reinterpretación temporal del «ser-en» que corrobora la temporalidad como el fundamento del cuidado, Heidegger señala que para que la comprensión y la disposición afectiva, en general, para que cualquiera de los elementos que conforman la aperturidad en general se temporice, cada uno de ellos debe estar co-originariamente determinado por los tres éxtasis, aunque Heidegger señala que primariamente lo sea bajo uno de ellos. Así, por ejemplo, y en relación a la temporalidad de la comprensión, Heidegger nos dice que dentro de una estructura co-originaria puede haber una preeminencia de alguno de sus elementos: «El comprender... es primariamente venidero. Pero no se temporizaría si no fuese tempóreo, es decir, si no estuviese co-originariamente determinado por el haber-sido y el presente» (SZ §68-a, 337). Del mismo modo, la disposición afectiva se temporiza primariamente desde el haber-sido, pero precisamente porque se temporiza, su éxtasis también pertenece co-originariamente al futuro y al presente bajo la modificación de este haber-sido (SZ §68-b, 340).

Sin embargo, algunos pasajes muestran claramente que, en conjunto con esta unidad horizontal-extática, hay dentro del planteo de la temporalidad cierta preeminencia del éxtasis futuro, el cual implicaría a su vez una primacía del momento estructural proyectivo de la comprensión dentro de la estructura del cuidado. En apoyo a esta posición citamos las propias palabras de Heidegger: 
El futuro tiene una primacía en la unidad extática de la temporalidad originaria y propia, si bien es cierto que la temporalidad no surge por adición y sucesión de éxtasis, sino que se temporiza siempre en la co-originariedad de los mismos (SZ §65, 329).

Marion Heinz, referente en las lecturas sobre la cuestión del tiempo en Heidegger, afirma en su artículo «The Concept of Time in Heidegger's Early Works» ${ }^{22}$ que la prioridad del futuro y su extenderse (Erstreckung) determinante sobre el haber-sido señala el hecho fundante de que para el Dasein todos los modos de ser son posibilidades ellas mismas y que, por lo tanto, todo lo que el Dasein ya ha sido debe ser incluido de un modo completo en este ser-posible. En este sentido, encontramos afirmaciones claras en Metaphysische Anfangsgründe der Logik, por ejemplo, al afirmar que el «haber-sido sólo «es» en cada caso de acuerdo con el modo de temporalización del futuro» (MAL § 12, 267).

Volviendo ahora, y finalmente, a la temporalidad específica de la disposición afectiva y, en particular, la patente en el temple anímico de la angustia, Heidegger aclara tal como mencionamos que la misma se funda primariamente en el haber-sido que consiste en llevar al Dasein de vuelta a la condición de arrojado como posibilidad repetible (SZ §68-b, 343). En esta primacía del haber-sido radica la posibilidad de modificar los otros dos éxtasis co-originarios de este estado de ánimo que representa la manera como cada vez yo soy primariamente el ente arrojado: el futuro y el presente como el estar retenido en el volverse a la más propia condición de arrojado y en este sentido, mantener al instante (Augenblick) de la resolución «a punto de producirse» (SZ §68-b, 344).

Pero, y en función de lo recién descripto, esto no debería ser incompatible con cierta preeminencia del futuro cuando comprendemos al temple anímico fundamental como elemento conformante del todo del cuidado. A esto mismo hace referencia Heidegger en el siguiente pasaje:

Si bien ambos modos de disposición afectiva, el miedo y la angustia, se fundan primariamente en un haber-sido, su origen, sin embargo, es distinto desde el punto de vista de la manera como cada uno de ellos se temporiza dentro del todo del cuidado. La angustia se origina a partir del futuro de la resolución; el miedo, desde el presente perdido (SZ §68-b, 344).

Heidegger nos está señalando que sólo se angustia genuinamente quien está resuelto, quien comprende que la angustia otorga la posibilidad de un poderser propio a partir de proyectos existenciales fundados en el éxtasis futuro. En este sentido, y siguiendo la recomendación del autor, debemos entender que, aun contemplando la primacía del haber-sido en relación a la disposición

[22] Heinz, M.: "The Concept of Time in Heidegger's Early Works"; en Kockelmans, J. ed.: A Companion to Heidegger's "Being and Time”. Washington: University Press of America, 1986; pp. 183207. También se puede acceder a una breve exposición sobre la preeminencia del futuro en la temporalidad del Dasein, consultando: Blattner, W.: Heidegger Temporal Idealism. Cambridge, New York: Cambridge Universtiy Press, 1999; pp. 116-118.

THÉMATA. Revista de Filosofía, №51 enero-junio (2015) pp.: 383-400 doi: 10.12795/themata.2015.i51.20 
afectiva de la angustia, y considerando su acento dentro de la multiplicidad de momentos que constituyen el ser-en, el mismo debe ser comprendido, dentro del todo del cuidado, como partiendo del futuro en el sentido recién descripto.

\section{Consideraciones Finales}

La lectura realista basada en el carácter desocultador de los temples anímicos se ha mostrado una hipótesis, aunque atractiva y probable, no exenta de dificultades. Aun considerando una función específica de los temples anímicos en el desvelamiento del mundo, no podemos admitir sin más aclaraciones su autonomía en relación a dicha tarea. Si bien los fenómenos cooriginarios de la aperturidad del Dasein son autónomos en el sentido de que no pueden deducirse unos de otros, tampoco pueden caracterizar cada uno de ellos por su lado el todo de la aperturidad.

La falta de separación entre la comprensión y la disposición afectiva (o, siguiendo la analogía propuesta por Hoffman, entre el conocimiento del ser y el conocimiento de lo sobrecogedor - del ente sin más), debe ser enmarcada en la finalidad general de Heidegger en esta época de acceder a la tematización del sentido del ser a partir de la interpretación fenomenológica del «ente que somos nosotros mismos», es decir, del Dasein. Esta mediación de la Analítica Existencial en relación a la pregunta fundamental se debe, según nos señala Vigo en su artículo "Temporalidad y Trascendencia", en primer lugar y ante todo, al hecho de estructural de que 'ser' es siempre, para Heidegger 'ser del ente'. Y esto debe ser comprendido en sus dos direcciones, a saber: que el ser es irreductible al ente (diferencia ontológica) y que el ser es inseparable del ente («inadmisibilidad de toda entificación por parte del ser»). ${ }^{23}$

Por último, consideramos que la relación de co-originariedad empleada en el análisis de los existenciales trabajados no es incompatible con la afirmación de cierta primacía de uno de los elementos conformantes de la estructura. Pero esta conclusión, lejos de devolvernos a la posibilidad de establecer una mayor autonomía en el carácter desocultador de los estados de ánimo, concluye dándole preeminencia al existencial de la comprensión, el cual ha sido ligado, tal como mencionamos, a las interpretaciones idealistas de Ser y Tiempo.

Lamentablemente, no podemos detenernos aquí en el análisis crítico de las lecturas idealistas de Heidegger basadas en la comprensión del ser. Baste decir, por el momento, que nuestro autor se muestra una vez más, y con el genio que lo caracteriza, como una fuente de estímulo inagotable a la hora de pensar estos problemas clásicos y de relevancia actual para el pensamiento filosófico.

[23] Vigo, A. G.: "Temporalidad y trascendencia, La concepción heideggeriana de la trascendencia del Dasein en Sein und Zeit" en Arqueología y aleteiología y otros estudios heideggerianos. Buenos Aires: ed. Biblos, 2008; pp. 39-58; p.40.

THÉMATA. Revista de Filosofía, No51 enero-junio (2015) pp.: 383-400 doi: 10.12795/themata.2015.i51.20 


\section{Referencias bibliográficas:}

Heidegger, M.: Metaphysische Anfangsgründe der Logik im Ausgang von Leibniz [MAL], Gesamtausgabe 26, Vittorio Klostermann, Frankfurt am Main, 1978.

: Principios metafísicos de la lógica; trad. Juan José García Norro, ed. Síntesis, Madrid, 2009.

: Prolegomena zur Geschichte des Zeitsbegriffs [PGZ], Gesamtausgabe 20, Vittorio Klostermann, Frankfurt am Main, 1979.

1963. : Sein und Zeit [SZ], Max Niemeyer Verlag, Tübingen,

Chile, 2000.

: Ser y Tiempo, trad. J. Gaos; FCE, Madrid, 1951.

: Ser y Tiempo; trad. J. Rivera; ed. Universitaria, Stgo de

: Vom Wesen des Grundes [WG], en Wegmarken, Gesamtausgabe 9, Vittorio Klostermann, Frankfurt am Main, 1976.

Blattner, W. D.: "Is Heidegger a Kantian Idealist?" en Dreyfus \& Wrathall ed.: Heidegger Reexamined / Vol. II: Truth, Realism and History of Being. New York, London: Routledge, 2002; pp. 231 a 248.

Blattner, W.: Heidegger Temporal Idealism. Cambridge, New York: Cambridge Universtiy Press, 1999; pp. 116-118.

Carman, T.: Heidegger's Analytic / Interpretation, Discourse and Authenticity in Being and Time. New York: Cambridge University Press, 2003.

Crowell, S.: "Facticity and Transcendental Philosophy" en Malpas, J. ed.: From Kant to Davidson. London, New York: Routledge, 2003; pp. 100 a 121.

Dreyfus, H. \& Spinosa Ch.: "Coping with Things-in-themselves: A Practice-Based Phenomenological Argument for Realism" en Dreyfus \& Wrathall eds.: Heidegger Reexamined vol. II / Truth, Realism and the History of Being. London, New York: Routledge, 2002; pp. 249 a 278.

Escudero, J. A.: El lenguaje de Heidegger/ Diccionario filosófico 19121927. Barcelona: ed. Herder, 2009.

Heinz, M.: "The Concept of Time in Heidegger's Early Works"; en Kockelmans, J. (ed.): A Companion to Heidegger's "Being and Time". Washington: University Press of America, 1986; pp. 183-207.

Hoffman, P.: "Heidegger and the Problem of Idealism" en Dreyfus \& Wrathall ed.: Heidegger reexamined / Vol. II: Truth, Realism and The History of Being. New York \& London: Routledge, 2002; pp. 319 a 327. 
Husserl, E.: Die Lebenswelt. Auslegungeng der vorgegebenen Welt und ihrer Konstitution (1929-1937) en Rochus Sowa ed.: Series Husserliana XXXIX. Dordrecht: Springer, 2008.

Inwood M.: A Heidegger Dictionary / The Blackwell Philosopher Dictionaries. Oxford: Blackwell Publishers, 1999.

Mulhall, S.: "Can There be an Epistemology of Moods?" en Dreyfus, Hubert L. and Wrathall, Mark A.: Heidegger Reexamined vol. 4 / Language and the Critique of Subjectivity. New York, London: Routledge, 2002, pp. 33 a 52.

Nussbaum, M. C.: Love's Knowledge / Essays on Philosophy and Literature. New York: Oxford University Press, 1990.

Olafson, F.: Heidegger and the Philosophy of Mind. New Haven: Yale University Press, 1987.

Vigo, A. G.: "Temporalidad y trascendencia. La concepción heideggeriana de la trascendencia del Dasein en Sein und Zeit" en Arqueología y aleteiología y otros estudios heideggerianos. Buenos Aires: ed. Biblos, 2008; pp. 39-58. 